\title{
qFit-ligand reveals widespread conformational heterogeneity of drug-like molecules in X-ray electron density maps
}

\author{
Henry van den Bedem \\ SLAC National Accelerator Laboratory, Stanford University, Menlo Park, CA 94025 USA
}

Structure-based drug design is often challenged by an inadequate understanding of the conformational dynamics of ligands and their receptors. X-ray-crystallography is generally the method of choice for resolving the structure of macromolecular molecules and investigating the binding pose of ligands. While the electron density represents a time-averaged representation of the underlying conformational ensemble, in the majority of cases the data are interpreted to represent a single conformation at unit occupancy.

B-factors, including anisotropic ADPs and TLS, offer a statistical validation of the data, but they inadequately depict deviations of atom positions from their averaged positions. Drug discovery, at the intersection of structural biology and computational chemistry, would benefit from statistically and biochemically validated, explicit representations of dynamic receptor-ligand binding poses.

qFit-ligand is a new, efficient and automated method for building parsimonious multi-conformer ligand models in electron densities by hierarchically sampling and building the ligand's degrees of freedom. We show that evidence for alternative ligand poses is common in the PDB, including for pharmaceutically highly relevant targets. qFit-ligand relies on powerful, state-of-the-art solvers to identify a minimal set of conformations to collectively explain the density and determine their occupancies.

We statistically and biochemically validated qFit-ligand multi-conformer models on a large benchmark data set of drug-like molecules. In a prospective study, we found that $29 \%$ of the BRD4 family of proteins in the PDB presented evidence of unmodeled alternative conformers of bound ligands. In many cases, these alternate conformations were adventitiously exploited to guide compound design, resulting in improved potency or selectivity. Accurate modeling of different binding poses for ligands will have important consequences for rational drug design and a fundamental understanding of the underlying binding mechanisms. 